Chronique des activités archéologiques de

\title{
Recherches archéologiques à Laos-Marcellina (Calabre, CS)
}

Campagne 2013

Vincenzo Capozzoli, Alain Duplouy et Alessia Zambon

\author{
(2) OpenEdition \\ Journals

\section{Édition électronique} \\ URL : http://journals.openedition.org/cefr/1145 \\ DOI : $10.4000 /$ cefr. 1145 \\ ISSN : 2282-5703 \\ Éditeur \\ École française de Rome
}

Référence électronique

Vincenzo Capozzoli, Alain Duplouy et Alessia Zambon, «Recherches archéologiques à Laos-

Marcellina (Calabre, CS) », Chronique des activités archéologiques de l'École française de Rome [En ligne], Italie du Sud, mis en ligne le 31 mars 2014, consulté le 01 mai 2019. URL : http://

journals.openedition.org/cefr/1145; DOI : 10.4000/cefr.1145

Ce document a été généré automatiquement le 1 mai 2019.

(c) École française de Rome 


\title{
Recherches archéologiques à Laos- Marcellina (Calabre, CS)
}

Campagne 2013

\author{
Vincenzo Capozzoli, Alain Duplouy et Alessia Zambon
}

1 En 2013, l'équipe franco-italienne a mené la cinquième et dernière campagne de prospection archéologique dans la basse vallée du $\mathrm{Lao}^{1}$. Nous rendrons donc compte ici de la seule prospection du territoire, coordonnée par Alain Duplouy (Université Paris 1), Priscilla Munzi (Centre Jean Bérard) et Alfonso Santoriello (Université de Salerne), aucune activité de fouilles n'ayant eu lieu cette année à l'intérieur du parc archéologique, et plus spécifiquement de l'activité de l'équipe française de l'Université Paris 1 PanthéonSorbonne.

2 L'activité s'est distribuée tout au long de l'année, selon les périodes de présence effective sur le terrain et les périodes d'étude en laboratoire. Outre les signataires, ont également pris part aux travaux de terrain ou de laboratoire Lucas Aniceto et Clément Salviani (doctorant et étudiant de master à Paris 1).

3 Comme annoncé lors de la précédente chronique, les travaux de cette année ont été consacrés, d'une part, à l'étude approfondie du matériel ramassé lors des saisons précédentes (2009-2012) et, d'autre part, à diverses vérifications ponctuelles sur le terrain. Nous avons du reste commencé l'étude d'un document d'archive tout à fait exceptionnel pour la topographie de la zone, déjà connu de nos prédécesseurs mais jamais publié.

\section{Vérifications de terrain}

4 Au cours du mois de septembre 2013, l'équipe française de l'Université Paris 1 a mené une brève campagne de vérifications sur le terrain en vue d'une meilleure compréhension de certains secteurs ayant livré des données qui se sont révélées intéressantes au cours de l'étude (fig. 1). 
Fig. 1 - Laos-Marcellina. Carte des UT prospectées ou vérifiées en 2013.

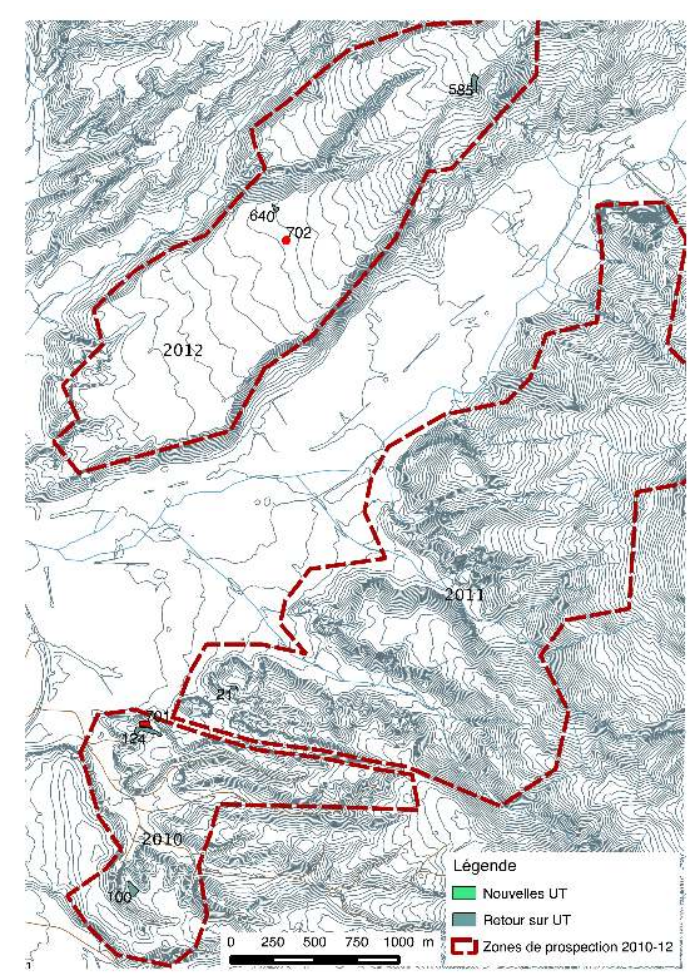

UMR 7041

5 En particulier, nous avons souhaité retourner sur les UT ayant livré du matériel céramique d'époque archaïque ou protohistorique (impasto). Le retour sur zone n'a jamais été inutile.

Il a tout d'abord permis de documenter plus amplement le mobilier d'époque protohistorique dans l'UT 21 (localité Marina) - dont nous avions trouvé quelques traces à l'occasion de la première campagne de prospection en 2009 et cherché en vain à les confirmer au cours des campagnes suivantes. Deux nouveaux fragments d'impasto y ont été ramassés, qui permettront de préciser la période de fréquentation de ce secteur.

7 Un retour sur la colline de Vitaliano, située immédiatement à l'est de la colline de San Bartolo où est implanté l'habitat lucanien, a permis de documenter plus amplement une indication bibliographique ancienne. Là, à proximité d'une petite église en ruine, E. Greco et F. G. La Torre rapportaient, sur la base de signalements de la Surintendance, la présence de nombreuses tuiles et plaques en calcaire, ainsi qu'une abondante céramique ${ }^{2}$. L'incendie d'un vaste terrain situé sur le plateau sommital a permis d'étendre quelque peu, par une nouvelle UT (701), les prospections menées là en 2009 (UT 19 et 20) et 2010 (UT 124-129). La découverte de nombreux fragments de tuiles corinthiennes, ainsi que de quelques tessons tend à confirmer les notices rapportées par La Torre et Greco. Ce matériel pourrait indiquer l'existence de tombes hellénistiques dans le secteur, dont certaines avaient été saccagées lors des travaux d'implantation de poteaux de l'ENEL dans les années 1970, mais la présence de couvre-joints (dont certains vraisemblablement de type pentagonal) pourrait aussi renvoyer à une structure d'habitat. 
De la même manière, un retour sur l'emplacement du site $n^{\circ} 132$ de G. F. La Torre, sur le sommet d'une petite colline au sud-est de San Bartolo, a permis de confirmer des informations anciennes. L'inspecteur y signalait la découverte de tessons et tuiles d'époque hellénistique à la suite d'une prospection réalisée en 1980, mais nous n'avions pu confirmer totalement ses indications en $2009^{3}$ et 2010 en raison d'une végétation abondante. En 2013, nous avons pu y retrouver plusieurs fragments de tuiles corinthiennes (UT 100), qui s'associent à divers couvre-joints de section semi-circulaire découverts en 2010 et renvoient ainsi à un système de couverture "hybride " caractéristique des périodes hellénistique et romaine dans toute l'Italie méridionale".

Sur la colline de la Suvareta, de l'autre côté du fleuve Lao, de nouveaux éléments ont été ramassés sur l'UT 640 (fragments d'amphores, de tuiles corinthiennes et de couvre-joints pentagonaux) qui viennent confirmer l'interprétation donnée l'année dernière sur l'existence d'un petit habitat rural hellénistique. À l'occasion de ce nouveau passage, une découverte fortuite (UT 702) a documenté un nouveau fragment de céramique commune à pâte claire et décor incisé "a pettine ", hors contexte mais qui vient recouper des indications déjà connues sur l'occupation du secteur au haut Moyen Âge ${ }^{5}$. Enfin, un retour sur l'UT 585, seule UT à avoir livré un fragment de vase archaïque dans cet ensemble de collines, n'a guère permis de compléter le tableau : tout au plus a-t-elle livré cette année quelques fragments de céramique fine, probablement à vernis noir mais au vernis usé.

\section{Un document cadastral de l'époque des Bourbons}

10 Lors de la mission de terrain effectuée en septembre 2013, l'équipe française de l'Université Paris 1 a poursuivi l'étude, entamée en 2010, d'un document d'archive conservé sous verre dans une collection privée à Marcellina. Il s'agit d'une grande carte (h. 1,54 m x l. 1,22 m), composée de 6 feuilles de papier assemblées, détaillant les terrains compris entre les vallées du Lao et de l'Abatemarco (fig. 2). 
Fig. 2 - Laos-Marcellina. Document cadastral du XVIII siècle.

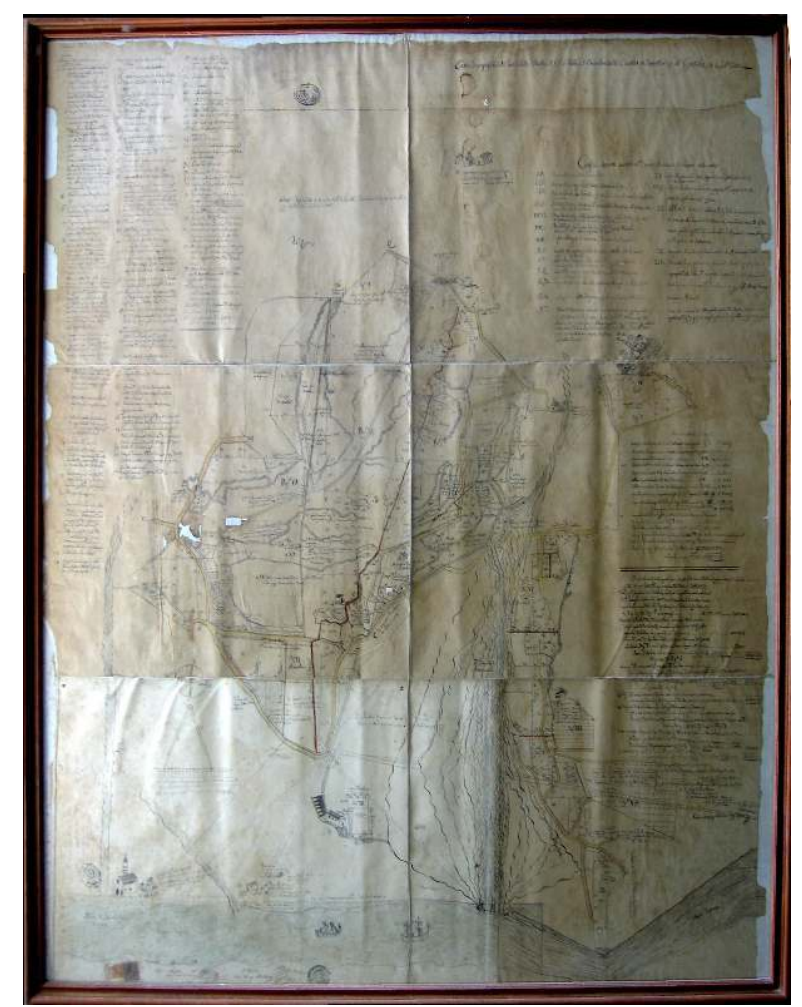

Collection privée, photo A. Zambon.

11 Elle est intitulée "Carta topograficha dei beni della Badia di S. Gio Batt[ist]a e S. Bartolomeo de' Cucutiis in territorio di Cipollina in Calabria Citra " et procède à un inventaire cadastral des biens de l'abbaye de San Giovanni Battista et San Bartolomeo de Cucutiis situés sur le territoire de la commune actuelle de Santa Maria del Cedro. Cette dernière, fondée au XVII ${ }^{\mathrm{e}}$ siècle, ne prit ce nom qu'en 1968 en relation avec le développement de l'économie du cédrat et du tourisme. Cipollina n'était du reste que l'appellation moderne du village nommé anciennement « Abatemarco » ou « Batemarco », dont l'implantation initiale correspondait d'ailleurs à l'emplacement du château de San Michele et de l'abbaye de San Giovanni Battista et San Bartolomeo sur un promontoire rocheux dominant la basse vallée du fleuve Abatemarco.

Le document est signé à deux endroits (au centre de la carte et en fin de description cadastrale): «Io, Notar Vincenzo Vicinoleg(at)o Agrimens(or)e faccio fede d'aver misurato e livellato $»^{6}$. Bien qu'il ne soit pas daté, la graphie autant que les conventions de dessin renvoient au XVIII ${ }^{\mathrm{e}}$ siècle. Divers éléments internes permettent en fait d'en situer la rédaction entre 1766 et 1775 (cf. infra). La carte elle-même a été vérifiée à plusieurs reprises; elle porte les mentions et les sceaux d'une vérification effectuée en 1850 et d'une autre, accompagnée de timbres fiscaux, en 1915.

13 La carte est dessinée à l'encre noire ; les principaux chemins sont coloriés en jaune, la mer Tyrrhénienne et les cours d'eau au crayon bleu. Quelques lignes tracées à l'encre rouge précisent les divisions cadastrales. Le nord est globalement situé sur le côté gauche de la feuille, comme l'indique une rose des vents ; la carte suit de bas en haut, soit d'ouest en est, le cours des fleuves Lao et Abatemarco. Elle présente une échelle graduée de 0 à 200, par unités de 10, avec l'indication: "Scala de' passi della quale sono state ancora 
regolate le distanze avvertendo che le medesime vi sono rimpicciolite, che ogni grado della scala contiene tre passi ». On notera néanmoins une certaine approximation dans le dessin des reliefs, des cours d'eaux, des chemins et des propriétés ; la carte n'a en aucun cas été levée selon des méthodes topographiques rigoureuses, comme la visée à partir de deux points remarquables du territoire, pourtant en vigueur à l'époque ${ }^{7}$.

Le territoire décrit est limité au nord (à gauche de la carte) par le fleuve Lao, nommé "Fiume Mercuro de comunem. di Scalea », et au sud (à droite de la carte) par le fleuve Abatemarco, nommé « Fiume di Batemarco ». La carte est centrée sur le village actuel de Marcellina, alors désigné comme " Marcellino ». Elle s'étend, de bas en haut, soit d'ouest en est, de la mer Tyrrhénienne à la source de plusieurs ruisseaux désignés comme « Fontana della Mortella » et « Fontana di Pietrarossa ». La carte représente les collines et vallons situés immédiatement à l'est de Marcellina. Elle retrace les axes viaires et chemins, les cours d'eau, les limites de propriété avec mesures et description. Elle présente aussi certains édifices remarquables comme les églises, les villages ou encore les ruines de l'agglomération ancienne de Batemarco situées autour du château de San Michele, désignée comme "Batemarco antico in cui vi sono trè famiglie ». D'autres édifices sont rendus en élévation: demeures ou hameaux (casino), chapelles, tours et moulins. Scalea, Ursomarso (i.e. Orsomarso) et Cipollina, par exemple, sont représentées par trois ou quatre maisons et une église avec son clocher. Détail pittoresque, deux galions naviguent le long de la côte dans la mer Tyrrhénienne.

Plusieurs légendes viennent compléter le dessin. En haut à gauche, une première liste, intitulée « Indice ", comporte 82 numéros correspondant à autant de points situés sur la carte. Cette liste donne des indications sur les toponymes ou sur la propriété des terrains, ainsi que sur la nature des cultures ${ }^{8}$. Une deuxième légende, située en haut à droite et constituée de 20 entrées, permet de circonscrire le territoire concerné par le document : "Confini descritti succintem(en)te poiché le contrade si relevano nella carta ». Des lettres majuscules de $\mathrm{A}$ à $\mathrm{Z}$, reportées sur la carte, forment des tracés, détaillés dans la légende, qui correspondent normalement à des rues ou à des limites de propriété. Une troisième liste, toujours sur la droite de la carte, intitulée "Estensione dei beni badiali », donne la description et les dimensions de douze entités géométriques (figure), correspondant à des ensembles de terrains, reportées sur la carte par des numéros ordinaux allant de I à XIII (même si l'entité VII manque tant sur la carte que dans la légende). Certaines de ces entités se composent de plusieurs sous-parties (porzioni), séparées par des lignes rouges. Une dernière liste, placée à la suite de la précédente, précise les termes du partage des biens de l'abbaye : «Divisione in tre parti egali in valuta de' detti beni Badiali principali prima del decreto ». Elle donne les valeurs monétaires en ducats des différents terrains et sous-parties composant les entités de la liste précédente.

Parmi les propriétaires féodaux ou les locataires des terres, deux en particulier retiendront ici notre attention, en ce qu'ils permettent de situer le document chronologiquement : il s'agit de Don Nicola Cavalcante et du Prince de Scalea. Ce dernier, mentionné aux numéros 74 et 75 en tant que propriétaire de terrains situés le long du fleuve Lao, fournit un premier repère chronologique. En effet, le fief d'Orsomarso, dont fait partie le territoire illustré ici, appartenait à la famille Brancati de Naples depuis $1668^{\circ}$ , mais ce n'est qu'en 1730 que la partie du fief située au-delà du fleuve Lao - c'est-à-dire au sud de celui-ci - fut acquise à Antonio Brancato par Francesco Spinelli (1681-1752), prince de Scalea ${ }^{10}$. Quant à Don Nicola Cavalcante, mentionné aux numéros 45, 47 et 69, il s'agit du marquis de Verbicaro Nicola Cavalcanti (1705-1775), à qui le fief d'Orsomarso fut 
donné en location à partir de 1766. Cette année constitue donc un terminus post quem pour la réalisation du document, tandis que la mort du personnage fournit un terminus ante quem. Le prince de Scalea mentionné dans la carte est donc le $8^{\mathrm{e}}$ du titre, Antonio II Spinelli (1715-1787) ${ }^{11}$. Des recherches en cours à l'Archivio notarile de Cosenza sur le notaire Vincenzo Vicino, auteur de la carte, devraient permettre d'en savoir davantage sur le contexte de réalisation du document.

17 Au-delà de l'histoire moderne, ce document fournit aussi de précieux renseignements à l'enquête archéologique. L'ensemble fera l'objet d'une analyse approfondie dans une étude ultérieure. Nous nous contenterons ici de quelques observations.

Tout d'abord, il est clair que le village de Marcellina existait déjà au XVIII ${ }^{\mathrm{e}}$ s., même s'il s'est progressivement urbanisé à la suite de la création de la station ferroviaire de Marcellina-Verbicaro-Orsomarso à la fin des années 1920. Le village possédait même, dès cette époque, une configuration analogue à l'agglomération actuelle, qui se présente sous la forme d'un triangle. Sa pointe occidentale (en bas sur la carte) correspond à peu de chose près à l'emplacement de la gare, tandis que sa base orientale longe le pied de la colline de San Bartolo (« via pubblica sopra Marcellino, e termine d'esso »), autrement dit les murailles antiques, sans pour autant qu'elles soient signalées. En outre, le tracé des axes viaires dans et autour du village correspond globalement à la configuration des principales routes modernes, bien qu'il soit impossible de les superposer puisque la carte n'est pas établie selon les principes de la cartographie scientifique.

La colline de San Bartolo ( $n^{\circ} 58$ ) parait elle-même vierge de toute implantation; aucune indication autre que le toponyme n'y figure: «Salso [i.e. alto] piano della Pronia osia Pironia che oggi chiamano di S. Bartolo»; la légende précise que ces terres étaient couvertes de vignes « d'uve durache ». Ce type de raisin était séché et connaissait un réel succès sur les marchés intérieurs et extérieurs, jusqu'à attirer navires anglais, hollandais et danois dans les ports de Scalea, Cirella et Diamante, comme le racontent les chroniques locales ${ }^{12}$.

Un seul élément de la carte correspond sans doute à des vestiges de l'Antiquité. Il s'agit de l'emplacement d'un « porcile » ( $\left.{ }^{\circ}{ }^{52}\right)$, dont la description fait référence, dans la légende, à un «muraglio ». Or, son emplacement correspond exactement à la localisation d'un tronçon de muraille de la ville lucanienne relevé par P. G. Guzzo et reporté sur le plan topographique de la colline, tronçon que les villageois nous ont encore indiqué le long de l'actuelle via Verbicaro en face du bowling (cf. les témoignages modernes rapportés dans Laos 2012).

21 Dans une approche à rebours du paysage antique de Laos, ce document cadastral de l'époque des Bourbons fournit un ensemble de renseignements qui pourront nous aider à mieux comprendre l'histoire, la topographie, la toponymie, ainsi que l'économie modernes de ce secteur compris entre le Lao et l'Abatemarco.

Le programme de prospection soumis à la Surintendance est désormais terminé. Les prochaines campagnes seront consacrées à la préparation d'une publication. 


\section{BIBLIOGRAPHIE}

Celico 2000 = G. Celico, Scalea tra duchi e principi, mercanti, filosofi e santi, Catanzaro, 2000.

Celico 2006 = G. Celico, L'oro dolce di Abatemarco, Cirella e Diamante nel XVIII secolo, dans L'Olmo, 43

(mars 2006), p. 7.

Cosenza 1981 = C. R. Cosenza, Esempio di un piccolo feudo nell'alto Tirreno cosentino nel Settecento, dans A. Placanica et A. Carvello (éd.), La Calabria dalle riforme alla restaurazione. Comunicazioni. Atti del VI Congresso storico calabrese [Catanzaro 29 ottobre- $1^{\circ}$ novembre 1977], II, Salerne-Catanzaro, 1981, p. 95-101.

Covino 2013 = L. Covino, Governare il feudo. Quadri territoriali, amministrazione, giustizia. Calabria Citra (1650-1800), Milan, 2013.

Girri 1758 = F.-M. Girri, L'agrimensore istruito, Venise, 1758 .

Greco 1995 = E. Greco (éd.), Città e territorio nelle colonie greche d'Occidente : Laos, Tarente, 1995.

La Torre 1999 = F. La Torre, Blanda, Lavinium, Cerillae, Clampetia, Tempsa : Lucania et Bruttium I, Florence, 1999.

Laos $2009=$ G. Aversa, A. Duplouy, V. Nizzo et A. Zambon, Recherches archéologiques à Laos-

Marcellina, dans MEFRA, 122-1, 2010, p. 320-330.

Laos 2010 = V. Amato, G. Aversa, L. Cavassa, A. Duplouy, F. Filocamo, P. Munzi, A. Santoriello, F. Uliano Scelza, A. Zambon, Recherches archéologiques à Laos-Marcellina, dans MEFRA, 123-1, 2011, p. 313-321.

Laos $2011=$ G. Aversa, A. Duplouy, P. Munzi et A. Santoriello, Recherches archéologiques à LaosMarcellina (Calabre, CS) : campagne de fouilles 2011, en ligne http://cefr.revues.org/421.

Laos 2012 = V. Amato, V. Capozzoli, L. Cavassa, A. Duplouy, F. Filocamo, P. Munzi, A. Santoriello, F. Uliano Scelza, A. Zambon, Recherches archéologiques à Laos-Marcellina (Calabre, CS) : campagne de fouilles 2012, en ligne http://cefr.revues.org/966.

Saint-Aubin 2003 = J.-P. Saint-Aubin, Une histoire du relevé, dans Géomètre, 1, 2003, p. 11-17.

Zambon 2011 = A. Zambon, Une leçon de topographie par correspondance : les lettres de l'ingénieur J. Foucherot et les levés du peintre L. F. S. Fauvel en Grèce, dans Le Monde des cartes, 207 (mars 2011), p. 107-124.

\section{NOTES}

1. Pour les campagnes précédentes, Laos 2009 ; Laos 2010 ; Laos 2011 ; Laos 2012. Comme chaque année, nous tenons à exprimer nos plus vifs remerciements à la Mairie de Santa Maria del Cedro, en particulier au maire G. Aulicino et au maire adjoint G. Pisciotti, pour le soutien qu'ils n'ont cessé de témoigner envers notre mission archéologique.

2. Greco $1995 \mathrm{n}^{\circ} 26$; La Torre $1999 \mathrm{n}^{\circ} 131$.

3. Laos 2009 , p. 329.

4. Laos 2012. 
5. Laos 2012.

6. Sur les « agrimensori » et leurs techniques de levé au XVIII ${ }^{\mathrm{e}}$ s., voir Girri 1758.

7. Sur les méthodes topographiques au XVIII ${ }^{\mathrm{e}}$ S., voir notamment Saint-Aubin 2003 et Zambon 2011.

8. Sur les cultures agricoles de la Calabre aux XVII ${ }^{\mathrm{e}}$ et XVIII ${ }^{\mathrm{e}}$ s., voir en dernier lieu Covino 2013.

9. Covino 2013, p. 81.

10. Cosenza 1981.

11. Sur le l'histoire médiévale et moderne de la région, voir en particulier Celico 2000.

12. Celico 2006.

\section{INDEX}

institutions Centre Jean Bérard (USR 3133 CNRS-EFR), Université Paris 1 Panthéon-Sorbonne (UMR 7041), Université de Salerne (Dipartimento di Scienze del Patrimonio Culturale), Soprintendenza per i Beni Archeologici della Calabria.

Mots-clés : prospection, archéologie du paysage, Lucanie, colonisation grecque

\section{AUTEURS}

\section{VINCENZO CAPOZZOLI}

Université Paris 1 Panthéon-Sorbonne, ArScAn (UMR 7041) - vincenzo.capozzoli[at]univ-paris1.fr

\section{ALAIN DUPLOUY}

Université Paris 1 Panthéon-Sorbonne, ArScAn (UMR 7041) - aduplouy[at]univ-paris1.fr

\section{ALESSIA ZAMBON}

Université Versailles-Saint-Quentin - alessia.zambon[at]uvsq.fr 